\title{
1. Extraction of Thermalized Projectile Fragments from a Large Volume Gas Cell
}

2 K. Cooper ${ }^{\mathrm{a}, \mathrm{b}}$, C. S. Sumithrarachchi ${ }^{\mathrm{a}}$, D. J. Morrissey ${ }^{\mathrm{a}, \mathrm{b}}$, A. Levand ${ }^{\mathrm{c}}$, J. A. Rodriguez $^{\mathrm{a}}$, G. Savard ${ }^{\mathrm{c}}$, S. Schwarz $^{\mathrm{a}}$, B.

\section{Abstract}

Experiments to determine the stopping and extraction efficiency of energetic $(90 \mathrm{MeV} / \mathrm{u}){ }^{76} \mathrm{Ga}$ fragments in a 1.2 meter long gas cell filled with helium at 123 mbar are reported. The thermalized ions were transported by DC and RF fields as well as gas flow, then jetted through a supersonic nozzle into a RF Quadrupole ion-guide and accelerated into an electrostatic beam line. The ions were collected in either a Faraday cup or a silicon beta-detector immediately after acceleration or after magnetic analysis. The range distributions of the ions and extraction efficiency were measured for different implantation rates and compared with the theoretically calculated values. The singly charged ${ }^{76}$ Ga ions were observed as $\left[{ }^{76} \mathrm{Ga}\left(\mathrm{H}_{2} \mathrm{O}\right)_{n}\right]^{+}$molecular ions with $\mathrm{n}=0,1$, and 2 . The stopping efficiency and the extraction efficiency were obtained from the measured distributions and compared to previous results from other devices.

Keywords: projectile fragments, thermalization

\section{Introduction}

Beam thermalization is a necessary process for the production of low-energy ion beams from high-energy projectile fragments. A number of systems have been developed to thermalize much lower energy recoils from nuclear reactions in gas beginning with the long-running IGISOL facility in Finland [1] and including the LISOL system in Belgium [2], the ShipTrap system at GSI in Germany [3], and the CPT and new CaRIBU system at Argonne National Lab (ANL) in the US $[4,5]$. The collection efficiency of devices for near-Coulomb barrier energies can be large, but the total efficiencies drop for energetic heavy ions due to large energy straggling. In addition, the ShipTrap system does not have to deal with high ionization rates. The present devices for thermalization of fast ions are located at RIKEN [6], GSI [7, 8], and the National Superconducting Cyclotron Laboratory (NSCL) [9, 10]. These devices generally use a linear gas-filled cylindrical chamber into which the beam enters along the central axis and the thermalized 


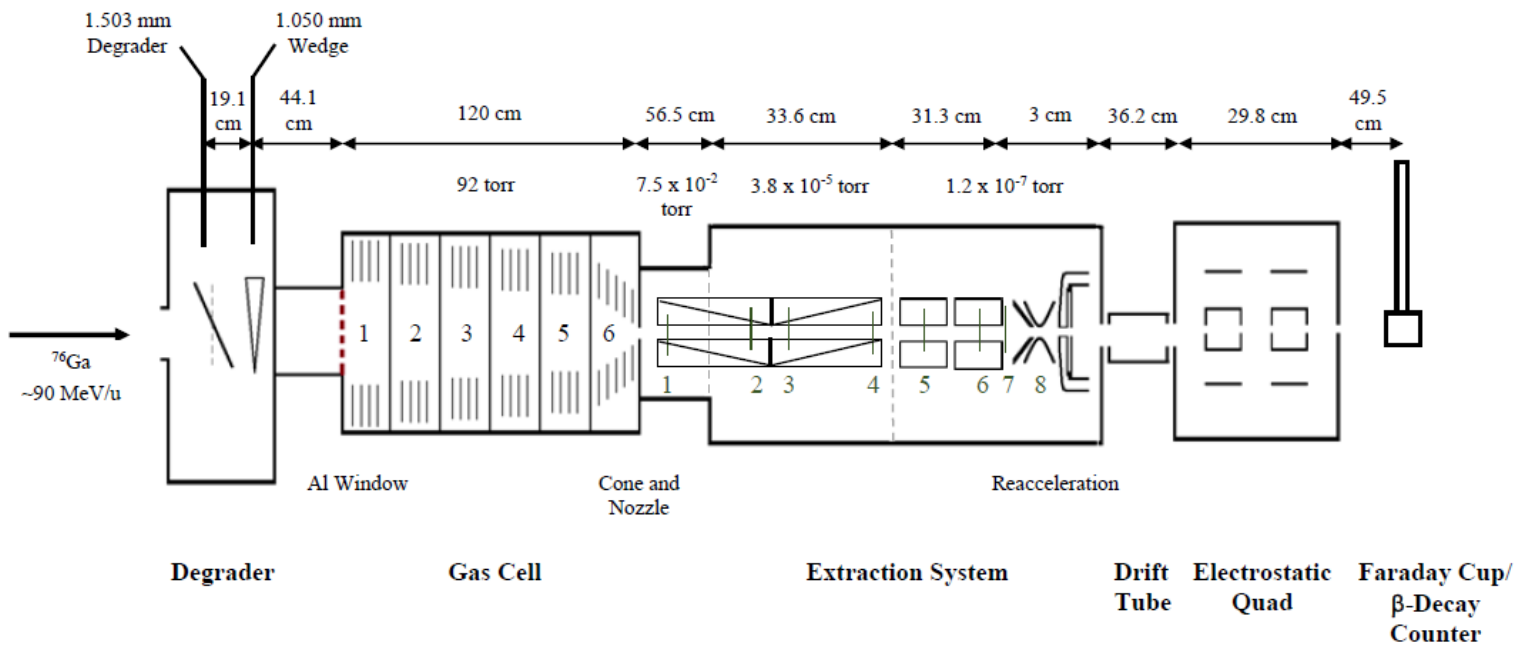

Figure 1: Schematic layout of the experimental equipment, not to scale. The distances between components are given in $\mathrm{cm}$.

ions are extracted on-axis at the far end of the cylinder. These devices also use electrodes in the gas to rapidly separate the more mobile electrons from the ionized buffer-gas atoms and the incident ions. A positive space charge builds up in the center of the chamber that tends to push the thermalized ions towards the walls and less-so towards the exit $[11,12,13]$. A new generation of devices have been designed with large internal volumes and RF ion-guiding structures along the walls of the chamber to attempt to mitigate these effects [5].

Recently, the NSCL upgraded its beam thermalization area with the installation of new high-resolution beam lines and a new large-volume RF-based gas catcher constructed by ANL. The high-energy projectile fragment beams $(\sim 100$ $\mathrm{MeV} / \mathrm{u}$ ) from the A1900 separator [14] are dispersed in momentum and then passed through solid degraders and a monochromatic wedge to remove nearly all their kinetic energy and to reduce the beam's energy spread, respectively, immediately before entering the 1.2 meter long gas catcher. Upon entering the chamber, the ions are thermalized through collisions with ultra-pure helium buffer gas. The high ionization potential of the helium gas forces most of the ions to remain ionized in the $1+$ or $2+$ charge state. The gas flow inside the chamber is relatively slow; therefore, a static drift field on the order of tens of $\mathrm{V} / \mathrm{cm}$ is used to sweep the positive ions towards the collection area where gas flow becomes strong enough to push the ions through the extraction orifice. A RF potential is applied to closelyspaced electrodes along the wall of the gas cell and along a RF cone that focuses the ions on the nozzle. Note that 
each incident projectile fragment will create $10^{5}$ to $10^{6}$ ion pairs in the helium gas during the slowing down process [15]. The RF potential repels the heavier ions from the electrodes along chamber walls directing them out of the cell while the very light $\mathrm{He}_{2}^{+}$and any $\mathrm{He}^{+}$ions are unimpeded and lost on the walls. The collection of the helium ions on the walls reduces both the space charge and the probability that they will charge-exchange with any impurities in the gas. The thermalized projectile fragments are jetted out into a new RFQ ion-guide [16] that maintains the ions on a central trajectory as they pass through three stages of differential pumping before acceleration to $30 \mathrm{keV} / \mathrm{q}$ and magnetic analysis. Several experiments were carried out to commission the new projectile fragment thermalization system using the ANL gas cell. Here we report the first results for the range distributions observed by the ionization of the buffer gas, the extraction of radioactivity as a function of degrader thickness, and the mass analysis of the extracted activities.

\section{Description of Experiment}

A primary ${ }^{82} \mathrm{Se}$ beam at $140 \mathrm{MeV} / \mathrm{u}$ was used with a $415 \mathrm{mg} / \mathrm{cm}^{2}$ beryllium target and a $299 \mathrm{mg} / \mathrm{cm}^{2}$ aluminum achromatic wedge located in the A1900 fragment separator [14] to produce a $75 \%$ pure $90 \mathrm{MeV} / \mathrm{u}^{76} \mathrm{Ga}{ }^{31}(\mathrm{~B} \rho=$ 3.4331 Tm) secondary beam. The primary beam current was frequently monitored by inserting a Faraday cup into the beam's path at the target position. A narrow momentum spread of $\Delta \mathrm{P} / \mathrm{P}=0.5 \%$ was used in the present work for the full acceptance of the separator. The only contaminant ion in the secondary beam was ${ }^{74} \mathrm{Zn}^{30+}$. A schematic diagram of the experimental setup is shown in Fig. 1. Most of the kinetic energy was removed from the high-energy secondary beam with a degrader system that included a 1503(5) $\mu \mathrm{m}$ thick Al plate and a silicon dioxide (glass) monoenergetic wedge that was $1050(50) \mu \mathrm{m}$ thick in the middle with a 5(0.9) mrad wedging angle. The calculated effective thickness of the aluminum degrader plate (final degrader) was varied by remotely adjusting its angle in the beam's path. The relatively small momentum spread of the secondary beam $(0.5 \% \Delta \mathrm{P} / \mathrm{P})$ was reduced as the dispersed beam passed through the glass wedge. The slowed fragments passed through a 37(2) $\mu \mathrm{m} \mathrm{Al} \mathrm{window} \mathrm{with} \mathrm{a} \mathrm{Titanium} \mathrm{alloy} \mathrm{support}$ grid and into highly purified helium gas at less than $1 \mathrm{MeV} / \mathrm{u}$. The support grid had an $85 \%$ transmission efficiency. High purity helium gas $(99.999 \%)$ was passed through a Monotorr purifier and a gas regulator system that held the pressure inside the chamber at 123 mbar. The gas filled chamber and all the materials inside were constructed and 

prior to the experimental work. As shown below, the helium gas was found to contain a high level of water vapor.

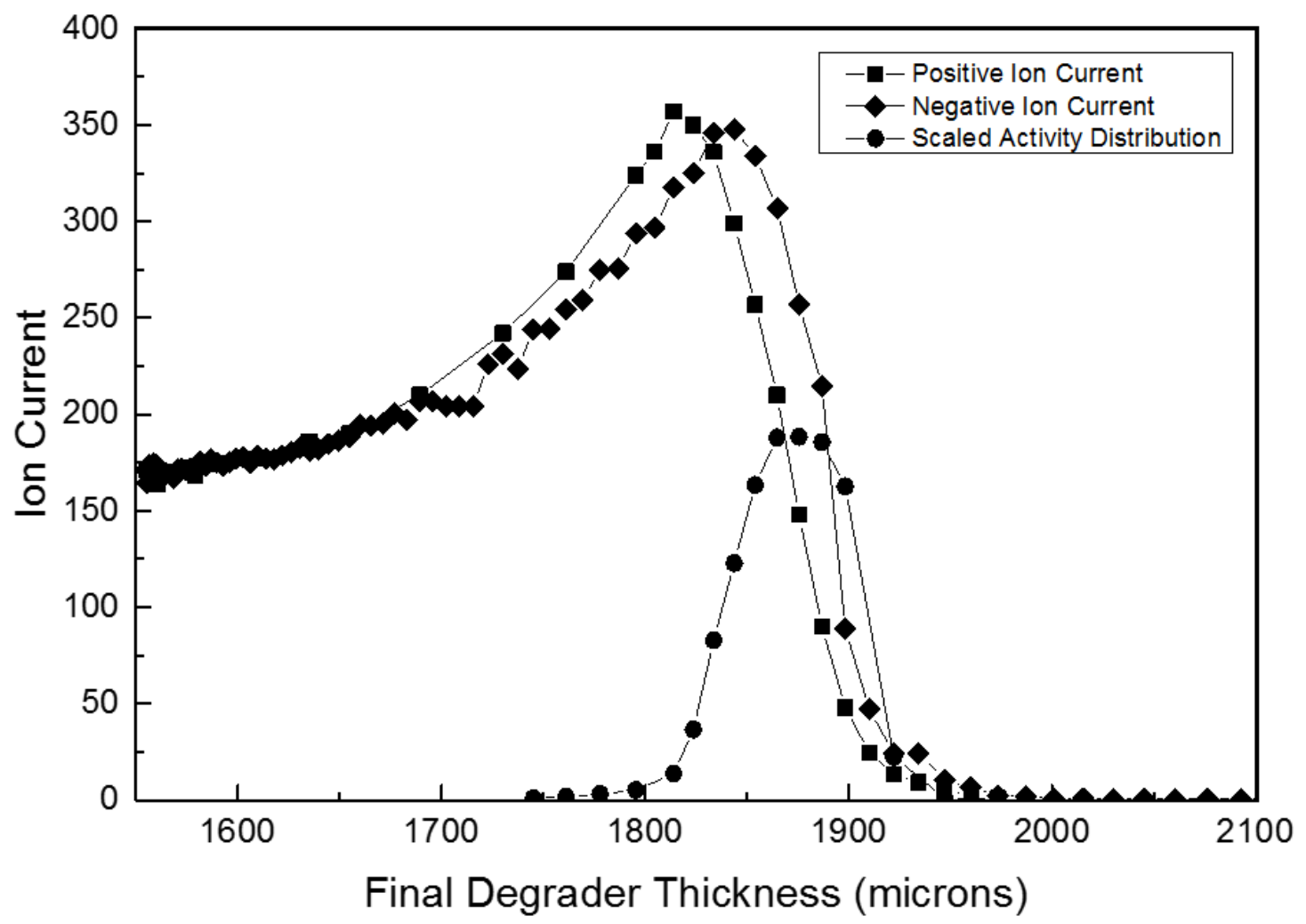

Figure 2: Measured distributions of the positive (squares, arbitrary scale) and negative (diamonds, nA) ion currents as a function of degrader thickness. The total scaled activity distribution measured with the first silicon detector (circles) is shown for reference. (Color Online)

A drift field of $7.5 \mathrm{~V} / \mathrm{cm}$ was applied with a bias of approximately $900 \mathrm{~V}$ between the entrance window (anode) of the gas catcher and the exit nozzle (cathode). Separate RF potentials were applied to the body and cone electodes in the cell at $3.514 \mathrm{MHz}(90 \mathrm{~W})$ and $3.180(50 \mathrm{~W})$, respectively. The electrode structures are described in [17]. The range distribution of the secondary beam was obtained by measuring (1) the total negative ion current on the window and (2) the total positive ion current on a Faraday cup after the ion-extraction system as a function of the effective thickness of the final degrader. The measured currents for an incident beam intensity of $\sim 7.6 \times 10^{5}$ pps agree within the uncertainty of the measurement and show the typical Bragg curve shape. A systematic shift in the value of the angle (thickness) between the two measurements was observed due to a mechanical lash. The total beta-decay activity 
(see below), also shown in Fig. 2, peaks near the midpoint of the falling curve as expected. The optimal angle for degrading the incident ions with the final degrader was determined to be $34^{\circ}(\sim 1876$ microns) from the maximum 71 yield of radioactivity.

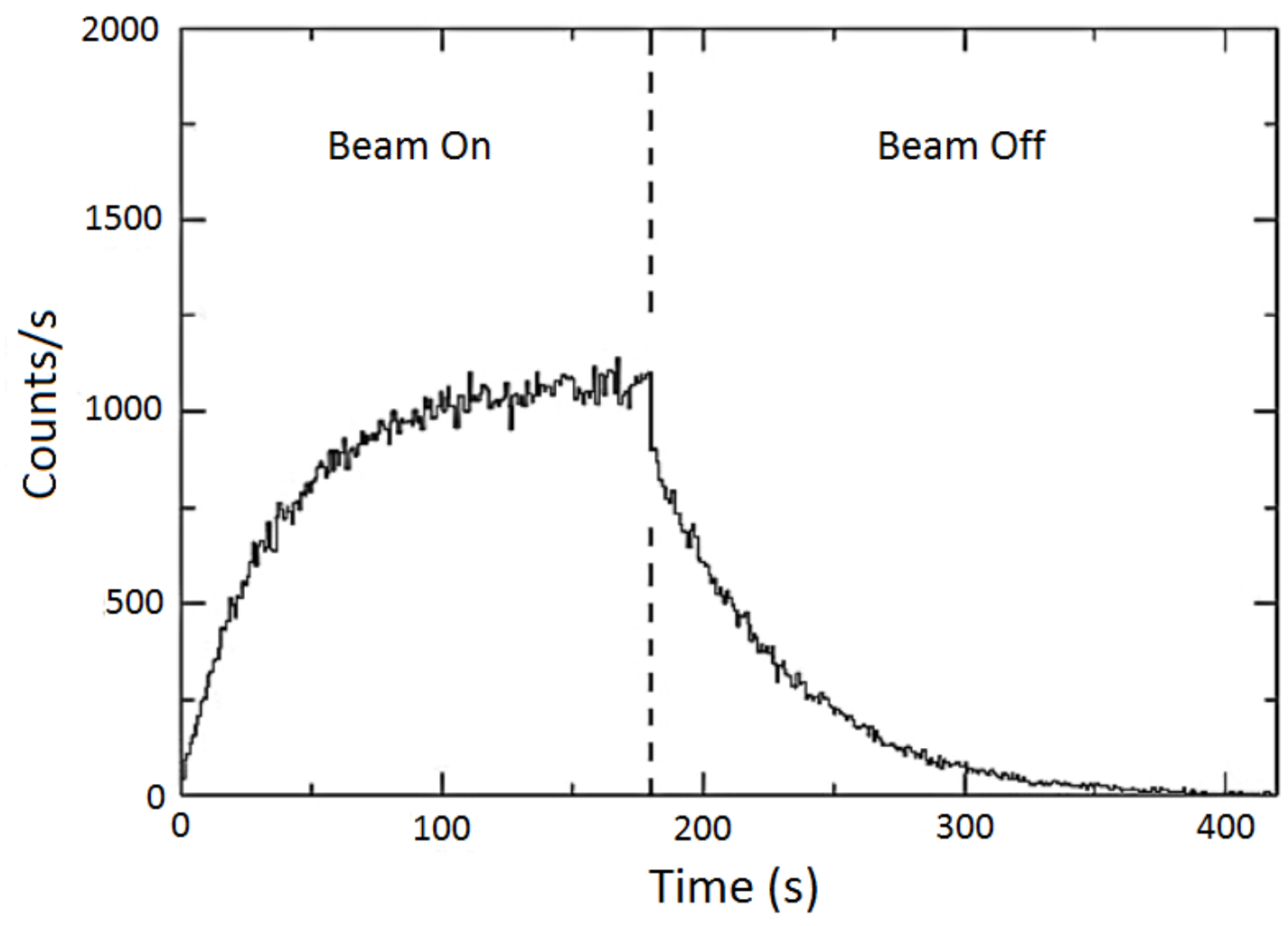

Figure 3: Example of the raw growth and decay curve of the total radioactivity observed at the exit of the extraction system when the degrader was tuned for the maximum radioactivity. The data are in very good agreement with the known half-life of ${ }^{76} \mathrm{Ga}$ which is $33 \mathrm{~s}$.

The thermalized projectile fragments were guided by the static DC and RF potentials applied to the gas cell's electrode structure towards the exit nozzle. The time needed for a typical atomic ion to drift half the length of the gas catcher under these conditions is approximately $40 \mathrm{~ms}$. The nozzle had a diameter of $1.3 \mathrm{~mm}$ and the total gas flow from the gas cell was measured to be $\sim 0.06$ standard-L/s. The ions were jetted through the nozzle into a differentially pumped expansion chamber and RFQ ion-guide [16]. The chambers in this section were evacuated by a three-stage differential pumping system to pressures of $7.5 \times 10^{-2}$ torr, $3.8 \times 10^{-5}$ torr, and $1.2 \times 10^{-7}$ torr respectively. The total radioactivity was collected on a thin foil 


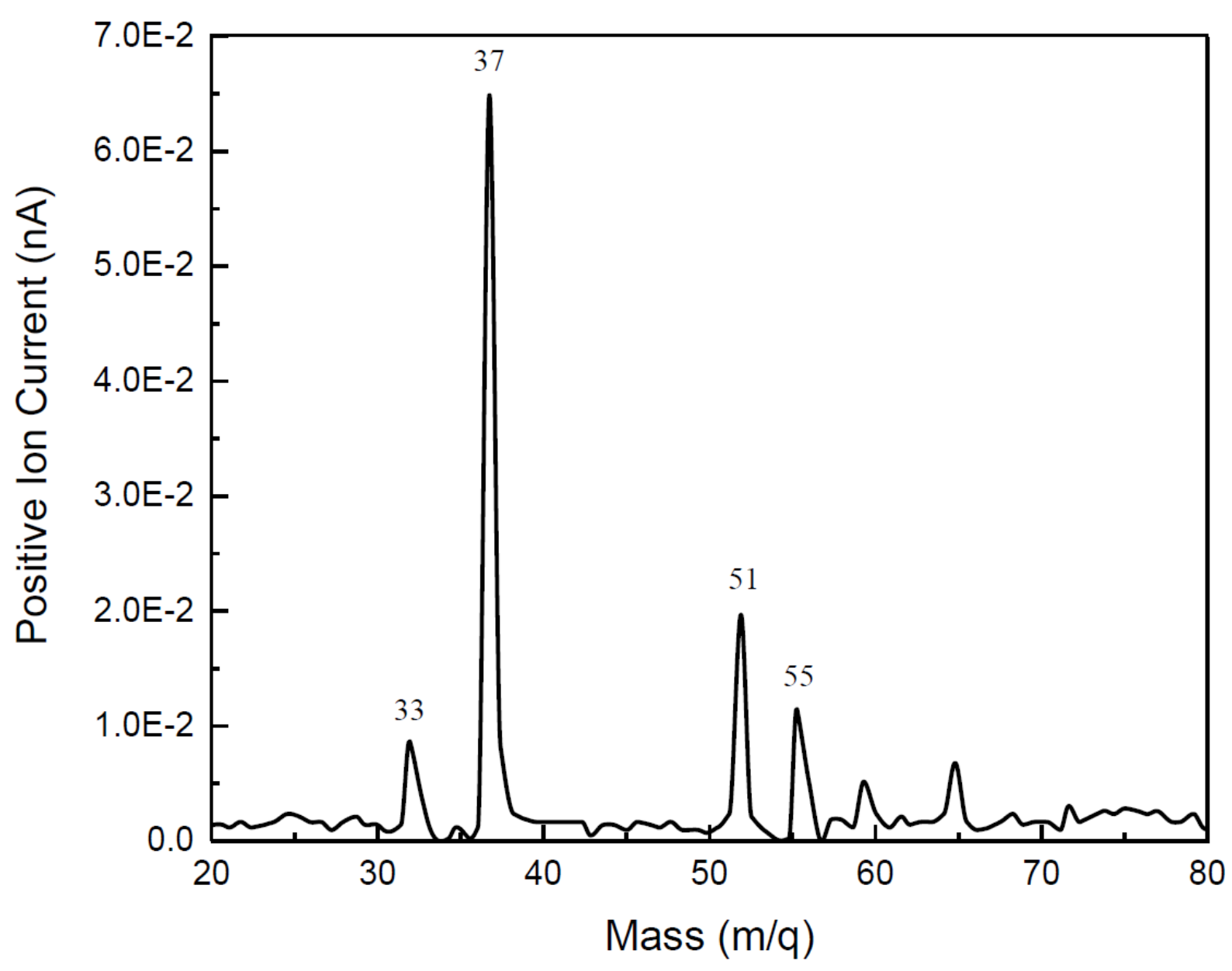

Figure 4: Part of the mass-to-charge spectrum of stable ions extracted from the gas cell observed in the Faraday cup with secondary beam entering the gas catcher. See the text for interpretation.

current and radioactivity could be measured as a function of final degrader thickness at the exit of the ion guides. The efficiency of the beta detector was determined using a ${ }^{90} \mathrm{Sr}$ source with a known strength to be approximately $33 \%$. An example of the growth and decay curve of the total activity is shown in Fig. 3.

The gas cell and extraction system were floated on a high-voltage platform at approximately $30 \mathrm{kV}$ to accelerate the ions into an electrostatic beam line to be transported to various experimental areas. The beam line contained a magnetic mass-analysis segment with a resolution, $M / \Delta M$, greater than 50 which sufficiently separated individual mass numbers in this range. A second silicon surface-barrier/Faraday cup device installed at the image position of the mass analyzer was used to measure the distribution of the extracted stable and radioactive ions. 


\begin{tabular}{cccc} 
Ion & $\begin{array}{c}\mathrm{m} / \mathrm{q} \\
\text { Decay Rate }\end{array}$ & $\begin{array}{c}\text { Fraction } \\
(\mathrm{cps})\end{array}$ & $(\%)$ \\
\hline${ }^{76} \mathrm{Ga}^{2+}$ & 38 & $<20$ & $<1(0.3)$ \\
${ }^{76} \mathrm{Ga}^{+}$ & 76 & 2070 & $24.3(0.6)$ \\
$\left.{ }^{[6} \mathrm{Ga}\left(\mathrm{H}_{2} \mathrm{O}\right)\right]^{+}$ & 94 & 2753 & $32.3(0.5)$ \\
$\left.{ }^{76} \mathrm{Ga}\left(\mathrm{H}_{2} \mathrm{O}\right)_{2}\right]^{+}$ & 112 & 2012 & $23.6(0.7)$
\end{tabular}

Table 1: Distribution of the observed ${ }^{76} \mathrm{Ga}$ activity among the molecular ions. See the text for details.

Figure 4 shows part of the mass-to-charge (m/q) spectrum of stable ions observed with the Faraday cup when the ${ }^{76} \mathrm{Ga}$ beam was entering the gas catcher. Peaks were observed at essentially all integral $\mathrm{m} / \mathrm{q}$ values. Recall that the chamber was not baked, and water can be expected to be present in the helium buffer gas. Prominent peaks in the $\mathrm{m} / \mathrm{q}$ spectrum at 37 and 55 are thus attributed to well known water adducts while peaks at 33 and 51 are attributed to the closely related $\left[\mathrm{CH}_{3}\left(\mathrm{H}_{2} \mathrm{O}\right)\right]^{+}$and $\left[\mathrm{CH}_{3}\left(\mathrm{H}_{2} \mathrm{O}\right)_{2}\right]^{+}$molecular ions. These molecular ions were created by charge transfer from the primary helium ions to neutral impurities in the gas that undergo subsequent chemical reactions with other water molecules before extraction. Similar water adducts were previously observed in the mass spectra from other gas cells, e.g., $[9,15]$.

The distribution of radioactivity after acceleration was scanned as a function of $\mathrm{m} / \mathrm{q}$ in the range of $\sim 30$ to $\sim 120$. The bulk of the activity was observed to be localized in three peaks. The values of the counting rates and the fractions of the total extracted activity for an incident beam rate of $7.3 \times 10^{3}$ pps are given in Table 1 . Approximately $20 \%$ of the total activity observed at the end of the ion guides was not observed in the mass-analyzed spectrum and was assumed to lie at higher mass values.

\section{Results and Discussion}

The measured Bragg curve was put onto an absolute scale and compared to the predictions of the TRIM Monte Carlo energy loss program that is part of the SRIM package [18]. The absolute current, I, in nA was converted into 
the energy loss in the helium gas of a single ${ }^{76} \mathrm{Ga}$ ion, $\Delta \mathrm{E}$, in $\mathrm{MeV} /$ incident-ion using the incident beam rate of $\mathrm{R}=$ $7.6 \times 10^{5}$ particles per second, the effective ionization value for helium gas of $\mathrm{W}=42 \mathrm{eV} /$ ion-pair, and the charge on the electron, $\mathrm{q}_{e}: \Delta \mathrm{E}=\mathrm{I} \mathrm{W} / \mathrm{R} \mathrm{q}_{e}$. The TRIM calculations used the beam momentum from the A1900 separator, its spread, and the total absorber thickness - thicknesses of the final degrader, the wedge, and the entrance window. The comparison between the simulated and experimental distributions agreed in both shape and magnitude; however, the simulated results had to be shifted up by $135 \mu \mathrm{m}$ (out of a total absorber thickness of $2973 \mu \mathrm{m}$ ). The comparison of the theoretically shifted values and the experimental data is shown in Fig. 5. The origin of the observed small, but significant shift is unknown at present but may be due to the fact that the magnetic rigidity of the A1900 fragment separator was never calibrated. Some error could also arise from nuclear reaction losses not accounted for by the TRIM simulation [19].

The yield of incident projectile fragments that are extracted from the gas catcher in the present work relies on two independent efficiencies: the fraction of thermalized projectile fragments stopped in the effective thickness of gas in the cell, $\epsilon_{S}$, and the fraction of thermalized projectile fragments that are extracted from the gas, $\epsilon_{E}$. Thus, the saturated activity observed in the detector after the extraction system, $\mathrm{A}_{s a t}$, is given by: $\mathrm{A}_{s a t}=\mathrm{R} \epsilon_{S} \epsilon_{E}$. The LISE++ program [20] was used to calculate the stopping efficiency for each degrader thickness and was corrected for the transmission through the window support grid. The stopping efficiency at the peak of the activity distribution was 0.48. The extraction efficiency was then obtained by dividing the saturated (total) radioactivity detected downstream of the extraction system by the rate of the incoming ${ }^{76} \mathrm{Ga}$ beam and the stopping efficiency. The extraction efficiency was evaluated for four different implantation rates ranging from $9.3 \times 10^{2}$ to $7.6 \times 10^{5}$ and determined to range from $34 \%$ to $16 \%$, respectively. These most recent results are in very good agreement with the previously established trends in extraction efficiency seen in a wide range of gas cells [12]. Equipment upgrades, including changes to the nozzle size and improvements in the vacuum system, are currently underway to further improve the extraction efficiency of the gas cell. 


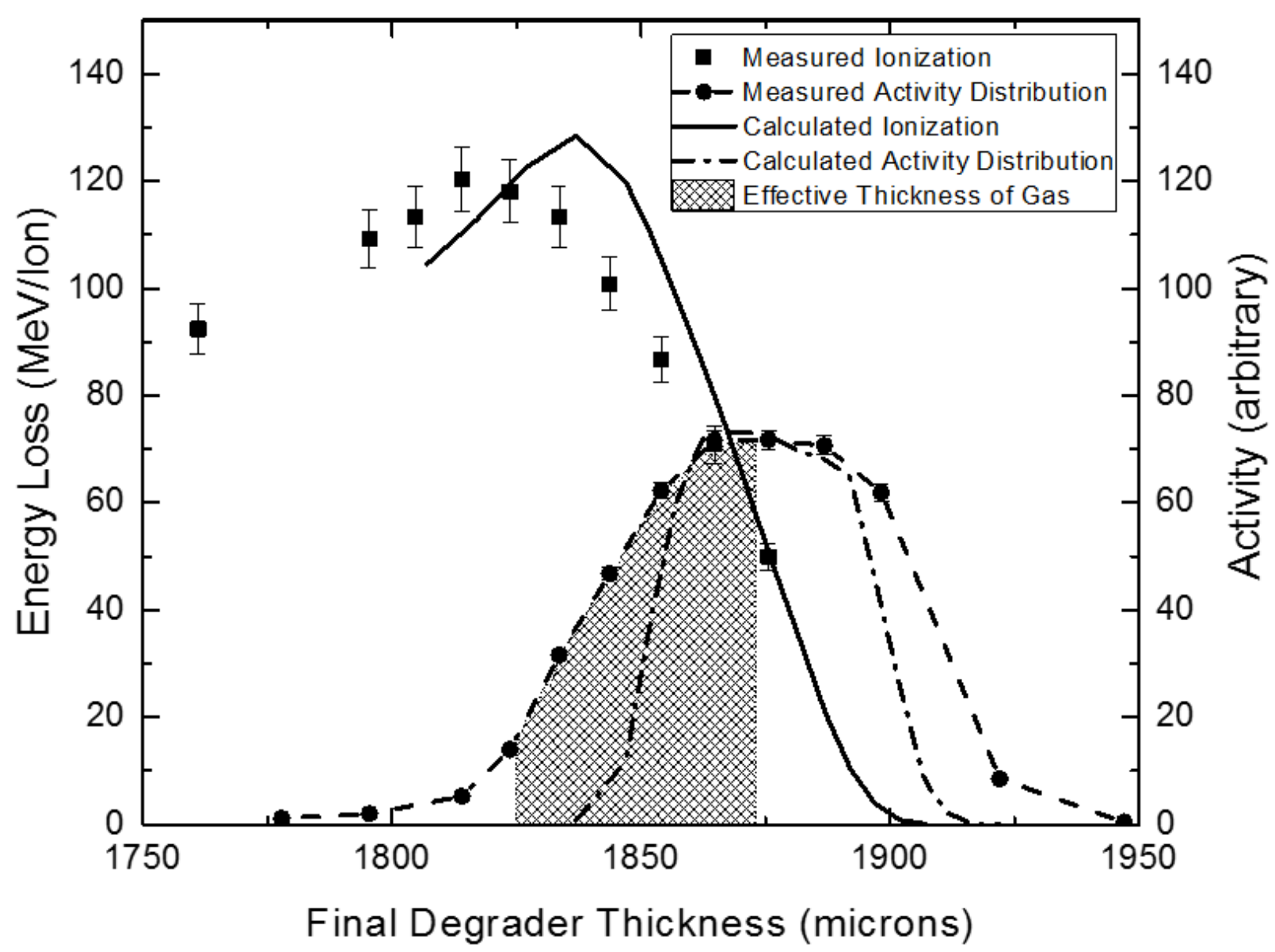

Figure 5: A comparison of the measured Bragg curve (squares) and the prediction from the TRIM code (solid line) as a function of degrader thickness is shown. Similarly, a comparison of the activity distribution of the radioactivity (circles with dashed line) with the same TRIM calculation (dash dot line) is also shown. The equivalent thickness of the helium gas is shown by the hatched area. (Color Online) See the text for details.

4. Summary

In summary, the successful operation of a new, large volume gas cell with RF electrodes along the entire inner volume and a focusing cone has been used to thermalize and extract a low-energy beam of projectile fragments. ${ }^{76} \mathrm{Ga}$ ions at $90 \mathrm{MeV} / \mathrm{u}$ were slowed in solid degraders and then thermalized and extracted from a gas catcher filled with helium gas at $\sim 120$ mbar. The total ionization and the extracted radioactivity were measured as a function of the particle's range, and the measured distributions agreed well with theoretical predictions. The extracted activities were mass-analyzed in a new low-energy beam line, and the bulk of the activity was found to be distributed among the bare 
ion and two molecular species. The extraction efficiency was found to be generally consistent with previous work.

\section{Acknowledgments}

We thank Scott Williams for his contributions to the data aquisition system. We also wish to acknowledge the support of Michigan State University, the National Science Foundation under cooperative agreement No. PHY-1102511, and the U.S. Department of Energy under contract DE-AC02-06CH11357.

\section{References}

[1] J. Aysto, et al., Eur. Phys. J. A 48 (2012) 42.

[2] Yu. Kudryavtsev et al., Nucl. Instr. Meth. B 266 (2008) 4368.

[3] S.A. Eliseev et al., Nucl. Instr. Meth. A 258 (2007) 749.

[4] G. Savard et al., Hyperfine Interactions 32 (2001) 223.

[5] G. Savard et al., Nucl. Instr. Meth. B 266 (2008) 4086.

[6] M. Wada et al., Nucl. Instr. Meth. B 204 (2003) 570.

[7] M. Petrick, et al., Nucl. Instr. Meth. B 266 (2008) 4493.

[8] S. Purushothaman et al., Eur. Phys. Lett 104 (2013) 42001.

[9] G. Bollen et al., Phys. Rev. Lett. 96 (2006) 152501.

[10] L. Weissman et al., Nucl. Instr. Meth. A 540 (2005) 245.

[11] A. Takamine, et al., Rev. Sci. Instrum. 76 (2005) 103503.

[12] D.J. Morrissey, Eur. Phys. J. Special Topics 150 (2007) 365.

[13] I. Moore, Nucl. Instr. Meth. B 266 (2008) 4434.

[14] D.J. Morrissey, et al., Nucl. Instr. Meth. B 204 (2003) 90.

[15] D.J. Morrissey et al., Nucl. Instr. Meth. B 266 (2008) 4822.

[16] B. R. Barquest, Ph.D. Dissertation MSU, 2014.

[17] G. Savard, J. of Phys: Conf. Series 312 (2011) 052004.

[18] The SRIM energy-loss package, J.F. Ziegler, http://www.srim.org/.

[19] J.F. Ziegler el al., Nucl. Instr. Meth. B 268 (2010) 1818.

[20] O.B. Tarasov and D. Bazin, Nucl Phys. A746 (2004) 411. 